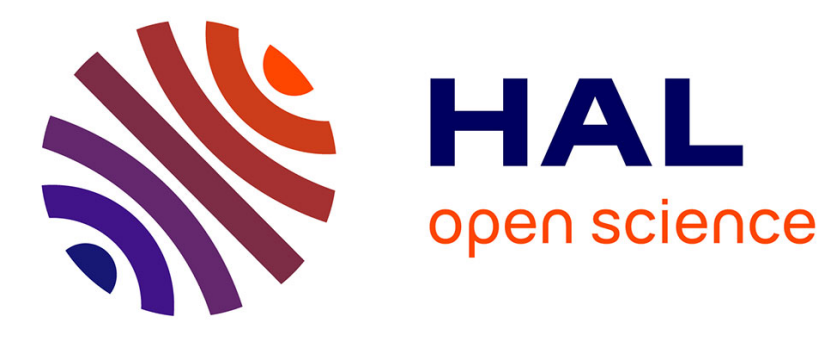

\title{
Vehicle Localization Integrity Based on Trajectory Monitoring
}

Olivier Le Marchand, Philippe Bonnifait, Javier Ibañez-Guzmán, David Betaille

\section{- To cite this version:}

Olivier Le Marchand, Philippe Bonnifait, Javier Ibañez-Guzmán, David Betaille. Vehicle Localization Integrity Based on Trajectory Monitoring. Intelligent Robots and Systems, 2009. IROS 2009., Oct 2009, St. Louis, MO, United States. pp.3453 - 3458, 10.1109/IROS.2009.5354156 . hal-00445124

\section{HAL Id: hal-00445124 \\ https://hal.science/hal-00445124}

Submitted on 7 Jan 2010

HAL is a multi-disciplinary open access archive for the deposit and dissemination of scientific research documents, whether they are published or not. The documents may come from teaching and research institutions in France or abroad, or from public or private research centers.
L'archive ouverte pluridisciplinaire HAL, est destinée au dépôt et à la diffusion de documents scientifiques de niveau recherche, publiés ou non, émanant des établissements d'enseignement et de recherche français ou étrangers, des laboratoires publics ou privés. 


\title{
Vehicle Localization Integrity Based on Trajectory Monitoring
}

\author{
Olivier Le Marchand ${ }^{1}$, Philippe Bonnifait ${ }^{2}$, Javier Ibañez-Guzmán ${ }^{1}$, David Bétaille ${ }^{3}$ \\ ${ }^{1}$ Renault SA, Guyancourt, France \\ ${ }^{2}$ Heudiasyc UMR 6599, Université de Technologie de Compiègne, France \\ ${ }^{3}$ Laboratoire Central des Ponts et Chaussées, Nantes, France
}

\begin{abstract}
For mobile robots to navigate autonomously, knowing with certainty their location is fundamental. However, this is difficult when operating in complex outdoors environments, and often results are inaccurate. To guarantee the certainty of the location estimates, the notion of integrity is used. This is a measure of the degree of confidence that provides a guaranteed location zone. The process consists in removing outliers amongst the measurements using Fault Detection and Exclusion (FDE) algorithms and then to compute Protection Levels (PL) that quantify the integrity zone. This is applicable mainly to Global Navigation Satellite Sytems (GNSS) in the aerospace domain where data redundancy exists and few multipath, which is not the case for land applications. In this paper, a new approach to localization integrity for land mobile robots is proposed by combining vehicle and GNSS data, stored within a short-term memory, the data horizon. This formulation allows for the application of FDE algorithms on the combined data set. It also leads to a gain of redundancy that enables PLs to be reduced and to increase the availability of integrity algorithms.
\end{abstract}

\section{INTRODUCTION}

Currently mobile robots are being successfully deployed in complex outdoors environments. As machines and individuals become interconnected multiple opportunities exist for Location Based Services (LBS). The feasibility of these applications depends very much on the accuracy of the localization systems, a critical issue for the successful deployment of vehicle navigation and safety related applications [1], [2].

When evolving outdoors the main source of absolute position information originates at GNSS, therefore when occlusion or signal multipath occurs, these result in inaccuracies. A thorough performance evaluation of GNSS receivers operating in standard traffic conditions has shown that the standard deviations recorded from the receivers do not reflect the true error of the vehicle trajectory [3]. As a result, assumptions with respect to the localization accuracy might be misleading. It is difficult to know when errors exist, this prevents the deployment of safety-related and location dependent vehicle navigation applications.

The concept of integrity has been of concern since the early 90s as aircraft navigation became more dependent on GNSS. It is defined as the measure of trust which can be placed in the correctness of the information supplied by the total system. It is an integral part of the Required Navigation Performance (RNP) formulation defined by the International Civil Aviation Organization for aircraft navigation purposes. The RNP includes concepts such as accuracy, integrity, continuity and availability to describe the safe navigation of vessels within a defined airspace.

To ensure the safe navigation of mobile robots, a similar approach could be adopted, by introducing the concept of integrity. Its availability will allow for deciding whether the position estimate is reliable for its intended use, and thus for the robot to decide if the location dependent applications can be performed.

Conventional approaches to integrity measurement rely on two algorithms: Fault Detection and Exclusion (FDE) to reject outliers from the measured data and the computation of the Protection Level (PL) associated to an integrity risk. These algorithms are originally suited to aerospace applications where high redundancy of data exists and faults are rare. In contrast, when navigating in urban environments, GNSS signals are occluded and thus there is seldom redundancy. Furthermore, multiple faults exists due to errors on the pseudo-ranges (estimated time of flight of the GPS signal, expressed in meters) originating from the multipath (reflection of GPS signals on the environment inducing a delay in the estimation). Typical localization systems for land robots, combine GNSS and dead-reckoning data. It shall be then possible to apply the Integrity concept to these data.

This paper proposes a new formulation of the localization problem, it combines vehicle ego-state information with GNSS data. It introduces the use of the data horizon concept, as a short term memory used to store measurement data, which allows for the use of standard integrity algorithms. FDE algorithms are applied on both GNSS and vehicle measurements due to the expression of their mutual redundancies through time. The PLs are decreased for the same reasons Finally, the redundancy gain allows for the application of integrity algorithms more often than when only relying on GNSS data.

The remainder of the paper is organized as follows: Section II presents FDE and PL algorithms developed in the aerospace domain for background purposes. Section III introduces the ground vehicle specificities found in complex urban environments applied to localization systems. The principles of the proposed formulation are described in Section IV. The application of the new formulation to passenger vehicles using GNSS based localization system is presented in Section V. The validation of the proposed approach is included in Section VI. A critique of the results and its applicability to navigation tasks are given in Section VII. 


\section{Fault Detection and Exclusion \& Protection LEVEL ALGORITHMS}

A fault is defined as "an unacceptable deviation of at least one characteristic property of the system from standard conditions" [4]. When the GPS is operating in ideal conditions, disturbances, such as multipath, are assumed to be non existent, the system is considered "fault free". For ground vehicles, multipath are considered as GNSS faults, as well as a wheel slippage when using wheel speed sensors. To remove these faults, FDE algorithms based on measurement redundancy are applied. Next, the degree of trust that can be placed in the location estimates is calculated. This is represented by protection levels (PL) [5]. This section outlines the computation of FDE for GNSS, Receiver Autonomous Integrity Monitoring (RAIM), and PL algorithm for background purposes.

\section{A. RAIM Algorithm}

This algorithm is applicable to any redundant measurement system. It is remarked that the presence of only one fault at a time is assumed. First it uses a least square resolution applied to a linearized observation equation:

$$
Y=h(X) \Rightarrow d Y=H . d X
$$

where $Y$ is the exteroceptive measurement vector (in GPS, pseudo-range vector for instance), $X$ is the state vector (in GPS, $X=\left[\begin{array}{llll}x & y & z & c d t\end{array}\right]$, that is the cartesian position and the receiver clock offset in meter), $d Y$ and $d X$ the respective linearized vectors. The noise on $Y$ is supposed to be independent, Gaussian and zero mean with known variance $Q_{Y} . H$ is the Jacobian of the observation function $h$ at the linearization point $X_{0}$ and is full rank. The estimated linearized state $d X$ is given by:

$$
d \hat{X}=\left(H^{T} Q_{Y}^{-1} H\right)^{-1} H Q_{Y}^{-1} \cdot d Y \hat{=} H^{+} \cdot d Y
$$

where $H^{+}$is the weighted pseudo-inverse of matrix $H$. The residuals $\varepsilon$ can be calculated as the difference between the measurements and the "estimated measurements" $h(\hat{X})$; these are derived from the estimated state:

$$
\varepsilon=Y-h(\hat{X}) \approx d Y-H d \hat{X}=\left(I-H H^{+}\right) d Y \hat{=} S \cdot d Y
$$

In normal conditions, the variable $d Y$ belongs to the kernel of matrix $S$, then the mean of each component of the residual vector is centered on 0 .

Now, if faults are added to the pseudo-range vector, Eq. 2 and Eq. 3 have to be rewritten:

$$
\begin{aligned}
d \hat{X} & =H^{+} \cdot(d Y+E) \\
\varepsilon & =S \cdot(d Y+E)
\end{aligned}
$$

where $E$ is the vector of faults. If only one fault of magnitude $b_{i}$ occurs on the $i$ th pseudo-range, i.e. only the $i$ th element of $E$ is non-zero and $E(i)=b_{i}$, then all the components of $\varepsilon$ are no longer centered on 0 . If no fault is present, the components of $\varepsilon$ follow a centered gaussian distribution verified with a $\chi^{2}$ test [6]: the Sum the Squared Error (SSE) has to be inferior to a threshold
Th equal to a $\chi^{2}$ distribution at $n-m$ degrees of freedom, having defined a probability of false alarm $P_{f a}$, where $n$ is the number of measurements used and $m$ the state size, $T h=\chi_{\left(1-P_{f a}, n-m\right)}^{2}$. This outlines the need of having many measurements $(n>>m)$ as it increases the degrees of freedom of the problem and makes it easier to perform detection. So, an integrity failure is detected if:

$$
S S E=\varepsilon^{T} Q_{Y}^{-1} \varepsilon>T h
$$

Once the occurrence of a fault is detected, it is necessary to identify the faulty measurement. Hence the score $w_{j}$ is computed for each measurement as a function of its residual and its variance (where $Q_{\varepsilon}$ is the residuals covariance matrix):

$$
\begin{gathered}
Q_{\varepsilon}=S \cdot Q_{Y} \cdot S^{T} \\
w_{j}=\left|\varepsilon_{j} / \sqrt{Q_{\varepsilon(j, j)}}\right|
\end{gathered}
$$

where the element of the ith row and jth column of a matrix $M$ is represented by $M_{(i, j)}$ in this paper. The maximum score indicates the faulty measure. This is then removed from the measurements vector, next a new position is calculated and a new detection test is performed and so on. It is observed that $w_{j}$ is a normalized score that takes into account both the geometrical configuration and the measurement noise thanks to the calculation of $Q_{\varepsilon}$. If FDE is applied to GNSS, it needs at least 5 satellites to perform detection, and 6 satellites to perform exclusion because the state size is equal to 4 . Even if the "one fault at a time" assumption is taken, multiple faults can be removed iteratively by this algorithm if the redundancy is sufficient regarding the correlation of faults [7].

RAIM algorithm underwent some improvement with adaptive threshold [8]. It is based on parity space methods which present similar results due to the linear formulation [6]. Nevertheless one necessary condition to apply these algorithms is the capability to represent the problem in a linear and observable manner as in Eq. 2. The performance comparison of these different methods for vehicle integrity is out of the scope of this paper.

\section{B. Protection Level Calculation}

Next the PL is computed as it represents a possible metric for localisation integrity. The concept of PL quantifies the maximum error in position that an undetected fault could cause. This is expressed in the positionning domain making it more comprehensible. It is often restrained to the xy error and called Horizontal Protection Level (HPL).

If a fault occurred only on the $i$ th measurement, with a magnitude equal to $b_{i}$, and computations are performed in a tangent local frame, then the error in the horizontal plane will be equal to :

$$
e_{\text {hor }}^{i}{ }^{2}=\left(H_{(1, i)}^{+}{ }^{2}+H_{(2, i)}^{+}{ }^{2}\right) b_{i}^{2}
$$

To link Eq. 9 to the norm of the residuals, the HSlope is defined. It depends on the geometrical configuration of the 
receiver with respect to the satellites, where $H_{S}$ Slope $_{M A X}$ represents the maximum $\mathrm{HSlope}_{i}$ for all measurements:

$$
e_{\text {hor }}^{i}=\sqrt{\frac{\left(H_{(1, i)}^{+}{ }^{2}+H_{(2, i)}^{+}{ }^{2}\right)}{\sum_{j} S_{(j, i)}^{2}}} \cdot\left\|\varepsilon_{i}\right\| \hat{=} \text { HSlope } i .\left\|\varepsilon_{i}\right\|
$$

Several formulas have been proposed to compute protection levels [5]. Most use the maximum of $\mathrm{HSlope}_{i}$ on all the satellites, multiplied by a value linked to the integrity parameters (previous $P_{f a}$ ). The Approximate Radial-error Protected (ARP) formulation is selected for this paper as the PL [6], which is defined as:

$$
A R P=\text { HSlope }_{\text {MAX }} \cdot T h
$$

More generally a probability of missed detection $P_{m d}$ can be considered for the PL computation. It takes in account through the computation of a minimum detectable bias, or the addition of the ellipsoid of the positioning uncertainty characterized by the $P_{m d}$, leading to the estimation of the HPL [9]:

$$
H P L=H \text { Slope }_{\text {MAX }} \cdot T h+R\left(P_{m d}\right)
$$

PL computation relies on the capability of expressing the observation problem with an invertible matrix, like in RAIM.

\section{Ground Vehicle Localization Specificities}

For ground vehicle localization, GPS is a key sensor as it is available worldwide. However, due to multipath occurrence in urban constrained environments, GPS positioning and RAIM performance are prone to severe deterioration. Unlike in open sky aerospace conditions, passenger vehicles encounter dense urban areas where streets are lined of buildings. The number of available satellites is often less than 7 [10].

Vehicle localization can benefit from vehicle specific sensors i.e. wheel speed and inertial sensors. It has been demonstrated that the combination of exteroceptive and proprioceptive sensors can significantly improve FDE. For instance, FDE based on an Extended Kalman filter (EKF) can perform the detection and exclusion of outliers in GPS measurements [11], [12] using innovation signals (known as pre-residuals). However, these techniques have been linked weakly to PL calculation. That is, the gain in outliers detectability has not been turned into a gain in the integrity metric. Actually, the estimation state from the update equation of the EKF is a function of the current measurements and the last estimated state, Eq. 13. Thus, the impact of a fault on the estimated state can be evaluated with an equation similar to Eq. 9, where $H^{+}$will be replaced by $K_{k}$. The estimated state can also be impacted by a biased predicted state due to faults occuring on the past measurements. To the authors knowledge, no formulation has been proposed to include such bias in the computation of PL.

$$
X_{k \mid k}=X_{k \mid k-1}+K_{k}\left(Y_{k}-H X_{k \mid k-1}\right)
$$

It is therefore proposed to consider data horizon in order to process a short term history of the trajectory, sometimes called finite memory approach.

\section{Trajectory Monitoring Algorithm}

\section{A. Data Horizon Principle}

This is one possible estimation method, it combines proprioceptive and exteroceptive data and has been used in diagnosis [13], [14]. The principle is to stack consecutive states and then to estimate them together. The method:

- Uses a discrete linearized model:

$$
\left\{\begin{array}{c}
X_{k}=A X_{k-1}+B P_{k} \\
Y_{k}=H X_{k}
\end{array}\right.
$$

- Stacks over time the states $X_{k}$ in one side, the measurement vector $Y_{k}$ and input vector $P_{k}$ on the other side. For instance, if matrix $B$ can be pseudo-inverted, stacking two states leads to Eq. 15:

$$
\left[\begin{array}{c}
Y_{k} \\
P_{k} \\
Y_{k-1}
\end{array}\right]=\left[\begin{array}{cc}
H_{k} & 0 \\
B_{k}^{+} & -B_{k}^{+} A_{k} \\
0 & H_{k-1}
\end{array}\right] \cdot\left[\begin{array}{c}
X_{k} \\
X_{k-1}
\end{array}\right]
$$

If the so composed matrix is full rank, then it can be pseudo-inverted, it is compatible with the formulation in Eq. 2. Hence the RAIM algorithm can be applied and the PL computed. However, this data horizon formulation presents a main drawback: it is impossible to separate input sensor failures from evolution model failures, because matrix $A$, which contains the evolution constraints, is involved in the expression of $P_{k}$. Hence a different formulation is needed to separate the evolution and input sensor models.

\section{B. Trajectory Observation Formulation}

Since we only address an observation problem, there is no need to use a state space formulation similar to Eq. 14 . In fact, proprioceptive measurements (usually placed in the input vector $P_{k}$ ) can be considered as observations of the traveled trajectory and the evolution model as constraints on it. As they measure or provide a priori information on the movement of the robot or its derivatives, they can be expressed as functions of the state derivatives in the continuous case or as functions of two or several consecutive states in the discrete case:

$$
\left\{\begin{array}{c}
\overrightarrow{0}=l\left(X_{k-q}, \ldots, X_{k}\right) \\
P_{k}=g\left(X_{k-r}, \ldots, X_{k}\right) \\
Y_{k}=h\left(X_{k}\right)
\end{array}\right.
$$

where $\overrightarrow{0}$ is a null vector and $g$ and $l$ are respectively the new expressions of the proprioceptive sensor model and of the evolution model with respect to the trajectory. By contrast to the previous Data Horizon method, this new formulation expresses the whole trajectory observation problem in a nonlinear manner, then linearization around a given trajectory gives:

$$
\left[\begin{array}{c}
\overrightarrow{0} \\
d \tilde{P} \\
d \tilde{Y}
\end{array}\right]=\left[\begin{array}{c}
\left.\frac{\partial l}{\partial X}\right|_{\tilde{X}_{0}} \\
\left.\frac{\partial g}{\partial \tilde{X}}\right|_{\tilde{X}_{0}} \\
\left.\frac{\partial h}{\partial \tilde{X}}\right|_{\tilde{X}_{0}}
\end{array}\right] . d \tilde{X}
$$




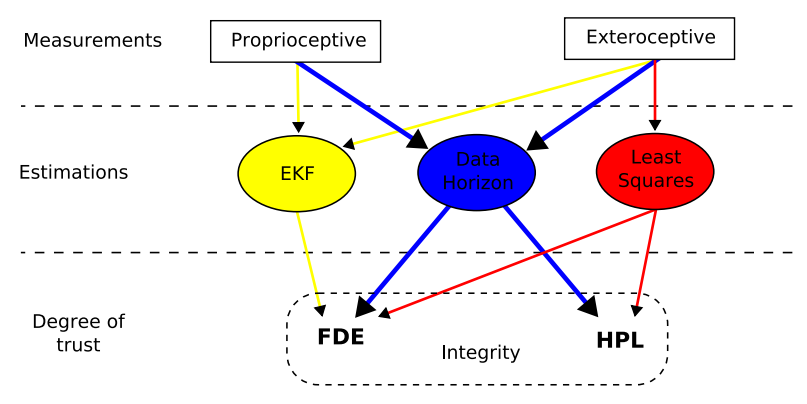

Fig. 1. Applicability of integrity to sensor types regarding data fusion schemes

where $\tilde{d P}, d \tilde{Y}, d \tilde{X}$ are respectively a linearized horizon of adapted depth of proprioceptive measures, exteroceptive measures and position. To compare with the previous formulation, stacking two states leads to:

$$
\left[\begin{array}{c}
Y_{k} \\
0 \\
P_{k} \\
Y_{k-1}
\end{array}\right]=\left[\begin{array}{cc}
H_{k} & 0 \\
\partial l / \partial X_{k} & \partial l / \partial X_{k-1} \\
\partial g / \partial X_{k} & \partial g / \partial X_{k-1} \\
0 & H_{k-1}
\end{array}\right] \cdot\left[\begin{array}{c}
X_{k} \\
X_{k-1}
\end{array}\right]
$$

Like in the previous formulation, RAIM algorithms can be applied and protection levels computed. The major difference is that FDE can be independently performed on the proprioceptive measurements $P_{k}$ and the evolution model (this being represented through constraints equal to 0 ). The protection level includes the whole trajectory stored in the data horizon, from which it is straightforward to extract the protection level of the current position (the last introduced in the buffer).

As represented in Figure 1, data horizon groups advantages of EKF and Least Squares together. Whilst the EKF allows the application of FDE to exteroceptive sensors with the help of proprioceptive sensors and Least Squares allows the application of FDE and HPL on exteroceptive measurements, the data horizon enables FDE and HPL computation on both exteroceptive and proprioceptive measurements. It matches an automotive integrity scheme.

\section{Theoretical Benefits from the Formulation}

As it extends integrity to proprioceptive sensors, the global redundancy is increased compared to FDE algorithms applied separately at each time step with Least Squares. This can be justified by comparing the degrees of freedom of this formulation to the sum of the degrees of freedom of individual GPS FDE. For example, if there are 6 satellites in view and a 4-size state, there are 2 degrees of freedom for a $\chi^{2}$ test of a RAIM algorithm. If a 4-samples data horizon is used with 2 proprioceptive sensors and one evolution constraint there are a maximum of 33 measurements $(6 \times 4+(2+1) \times 3)$ for a 16-size state, so there are 17 degrees of freedom compared to 4 times 2 degrees of freedom with individual FDE. This increase of redundancy enhances the performance of FDE algorithms and their use even when there are few exteroceptive measurements.

The impact of a fault on the horizontal positioning error, $e_{i}$, depends on its magnitude and on the weight of the measurement in the position estimation. With respect to a one epoch Least Squares, data horizon decreases the weight of each exteroceptive measurement at this epoch because they are linked to the exteroceptive measurements at previous epochs through the proprioceptive data and the evolution

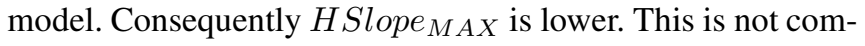
pensated by the $T h$ increase (due to the degree of freedom rise), so $A R P$ is reduced, as presented in Section VI.

The following section will present a test case.

\section{Application to Passenger Car Localization}

The formalized strategy based on Eq. 17 has been applied to the localization of a car vehicle equipped with two wheel speed sensors, a yaw rate gyroscope, a GPS receiver, and under the assumption of a constant altitude evolution model. For simplification this section presents only part of the developed model. The GPS model is the one used in tightly coupled solutions, details can be found in [12]. The state is expressed in an earth-tangential local frame using a Cartesian coordinates system. The location is augmented by the clock bias of the receiver for each epoch, leading to $X_{k}^{a}=\left[\begin{array}{llll}x_{k} & y_{k} & z_{k} & c d t_{k}\end{array}\right]^{T}$.

The car reference frame is defined at the center of the rear axle, the speed of a wheel (here the rear left at time $k: v_{r l, k}$ ) is a function of the distance traveled by the rear axle center corrected by the difference of speed between left and right wheel due to the turning maneuver:

$$
v_{r l, k}=g_{r l}\left(X_{k}^{a}, X_{k-1}^{a}, X_{k-2}^{a}\right)=\frac{\left(d_{k}-\frac{L\left(\theta_{k}-\theta_{k-1}\right)}{2}\right)}{\Delta t_{k}}
$$

where $d_{k}$ is the distance traveled between two epochs, $\theta_{k}$ the current heading, $L$ the width of the rear axle and $\Delta t_{k}=t_{k}-t_{k-1}$. Assuming a $2 \mathrm{D}$ motion in the horizontal plane and a sufficient sampling of the trajectory, $d_{k}$ and $\theta_{k}$, can be expressed as:

$$
\begin{gathered}
d_{k}=\sqrt{\left(x_{k}-x_{k-1}\right)^{2}+\left(y_{k}-y_{k-1}\right)^{2}} \\
\theta_{k}=\operatorname{atan} 2\left(\left(y_{k}-y_{k-1}\right),\left(x_{k}-x_{k-1}\right)\right)
\end{gathered}
$$

It is remarked that $v_{r l, k}$ is expressed as a function of $\theta_{k-1}$, it self depending on $x_{k-2}$ and $y_{k-2}$, thus it is defined as a function of three consecutive states. Then it can be derived:

$$
\begin{aligned}
& \frac{\partial d_{k}}{\partial X_{k}^{a}}=G_{k}^{d}=\left[\begin{array}{llll}
\frac{\left(x_{k}-x_{k-1}\right)}{d_{k}} & \frac{\left(y_{k}-y_{k-1}\right)}{d_{k}} & 0 & 0
\end{array}\right] \\
& \frac{\partial \theta_{k}}{\partial X_{k}^{a}}=G_{k}^{\theta}=\left[\begin{array}{llll}
\frac{-\left(y_{k}-y_{k-1}\right)}{d_{k}^{2}} & \frac{\left(x_{k}-x_{k-1}\right)}{d_{k}^{2}} & 0 & 0
\end{array}\right] \\
& \frac{\partial g_{r l}}{\partial X_{k}^{a}}=G_{k, 0}^{r l}=\frac{G_{k}^{d}+L \cdot G_{k}^{\theta} L \cdot G_{k}^{\theta} / 2}{\Delta t_{k}} \\
& \frac{\partial g_{r l}}{\partial X_{k-1}^{a}}=G_{k, 1}^{r l}=\frac{-2 G_{k}^{d}-L \cdot G_{k-1}^{\theta}-L \cdot G_{k}^{\theta}}{2 \Delta t_{k}} \\
& \frac{\partial g_{r l}}{\partial X_{k-2}^{a}}=G_{k, 2}^{r l}=\frac{L \cdot G_{k-1}^{\theta}}{2 \Delta t_{k}}
\end{aligned}
$$


The introduction of an evolution model can be done in the same way. For example, if the altitude has to be constrained as a constant (in order to improve bad stability of GPS altitude) a simple equation can be added:

$$
0=l_{d z}\left(X_{k}^{a}, X_{k-1}^{a}\right)=z_{k}-z_{k-1}
$$

An example of the full trajectory observation matrix with a 4-samples state horizon, a GPS sensor, a rear left wheel speed sensor, and a constant altitude constraint is given as:

$$
\left[\begin{array}{c}
0 \\
0 \\
0 \\
d v_{r l, k} \\
d v_{r l, k-1} \\
d \rho_{k} \\
d \rho_{k-1} \\
d \rho_{k-2} \\
d \rho_{k-3}
\end{array}\right]=\left[\begin{array}{cccc}
L^{d z} & -L^{d z} & 0 & 0 \\
0 & L^{d z} & -L^{d z} & 0 \\
0 & 0 & L^{d z} & -L^{d z} \\
G_{k, 0}^{r l} & G_{k, 1}^{r l} & G_{k, 2}^{r l} & 0 \\
0 & G_{k-1,0}^{r l} & G_{k-1,1}^{r l} & G_{k-1,2}^{r l} \\
H_{k} & 0 & 0 & 0 \\
0 & H_{k-1} & 0 & 0 \\
0 & 0 & H_{k-2} & 0 \\
0 & 0 & 0 & H_{k-3}
\end{array}\right]\left[\begin{array}{c}
d X_{k}^{a} \\
d X_{k-1}^{a} \\
d X_{k-2}^{a} \\
d X_{k-3}^{a}
\end{array}\right]
$$

where $\rho$ is the pseudo-range vector and:

$$
L^{d z}=\left[\begin{array}{llll}
0 & 0 & 1 & 0
\end{array}\right]
$$

This example provides a mathematical formalization of the data horizon approach.

\section{RESUlts}

The results of the experiments performed to validate the proposed approach are presented in this Section. They were designed to validate the FDE capabilities, and to identify the impact of the formulation on the PL regarding to the introduced vehicle dynamic and the data horizon depth.

For this purpose the trajectory of a passenger vehicle and ego-state variables were recorded together with data from an automotive-type GPS, in real traffic conditions. The egostates variables are extracted from the rear wheel speeds and a yaw rate gyroscope. The vehicle was driven for a distance of $2.5 \mathrm{~km}$ in an urban context. This data was processed offline and faults introduced on the pseudo-ranges to emulate their occurrence, as described in [12]. The introduction of the faults allows to run the algorithm with certainty of their existence. The objective is to take into account faults arising due to the presence of multipath on the GPS measurements. The FDE and PL algorithms are applied to this data set.

The results are shown in Figure 2, the vehicle travels in a straight line at $20 \mathrm{~m} / \mathrm{s}$. The GPS measurements are taken considering only the 6 higher satellites. The length of the data horizon chosen was 8 samples, which considers the vehicle response. To simulate the occurrence of faults, a bias of $15 \mathrm{~m}$ was introduced to the pseudo-range of the same satellite, for the last two samples. A false alarm probability of 0.001 was taken. The measurements on the true trajectory are represented by diamonds (green color). The circles (blue color) represent the positions estimated applying the data horizon based algorithms. The crosses (color red) are the positions estimated independently at each sample with the conventional RAIM algorithm applied to GPS data. The figure presents a typical behavior of an iterated RAIM algorithm applied to the proposed formulation when multiple faults exist. It can be remarked that the last two crosses have a larger error due to the fault introduced onto one of the pseudo-ranges. The FDE algorithm on the GPS only estimation can not detect the error due to the low bias value.

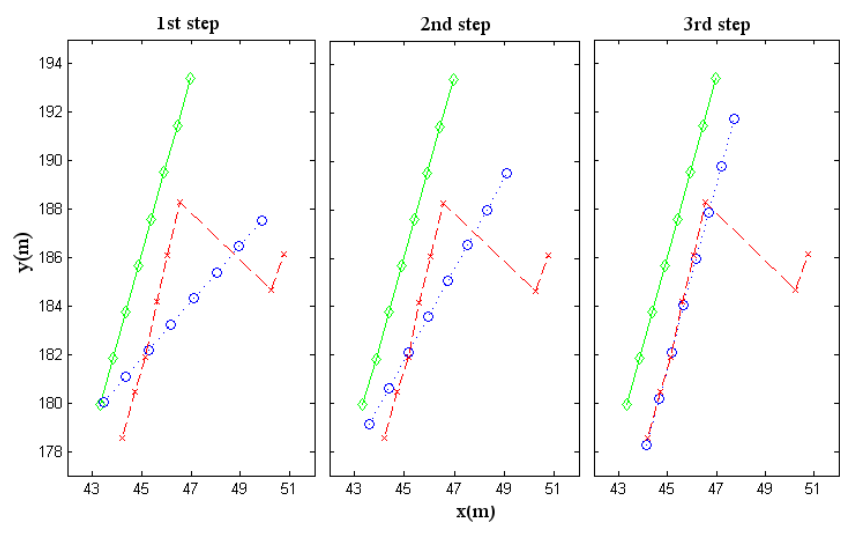

Fig. 2. Consecutive steps of data horizon based FDE

Figure 2, shows three steps that represent the consecutive use of the FDE as a results of the data horizon. The first step represents the estimation through the data horizon for all the measurements including the two biases. In the second step, an iterative RAIM algorithm is applied. This has led to the detection and removal of one of the two faults and to generate a new trajectory estimate. For the third step a new detection and exclusion stage is performed. This results in a further correction of the trajectory estimate as shown in Figure 2. It is important to note that the shape of the estimated trajectory using the new algorithm is continuous (no jumps) for all steps. The heading is corrected gradually, the alignment and the distance between points show the contribution of the proprioceptive sensors on the estimation.

From the previous trajectory and the satellite configuration at this time, the ARP was computed for the whole trajectory, taking into account 7 satellites for a data horizon depth of 8 samples. The results are shown in Figure 3. The variations in the ARP during the trajectory are due to the vehicle speed and yaw rotation. It is observed that a long straight line at $70 \mathrm{~km} / \mathrm{h}$ leads to the lowest ARP between $t=200 \mathrm{~s}$ and $t=$ $500 \mathrm{~s}$. This configuration is well-suited for reducing position uncertainty by benefiting of the position filtering. It allows for the reduction in the ARP. By contrast moving at low speeds with the vehicle in a turning maneuver (e.g. crossing a round about) leads to a high ARP, as what occurs between $t=0 \mathrm{~s}$ and $t=250 \mathrm{~s}$.

An interesting point is the impact of the horizon depth: the deeper the data horizon, the lower the ARP, as shown in Figure 4. It presents the ARPs computed for different horizon depths. The contributions of the measurements by the proprioceptive sensors and evolution models to express the temporal redundancy, as described in Section IV, can be there observed. Further, the gain is non-linear with regard to the data horizon depth, and it seems to reach a limit: adding old measurements provides less and less information.

Further tests will be performed in order to assess the performance of the new formulation regarding to usual FDE 


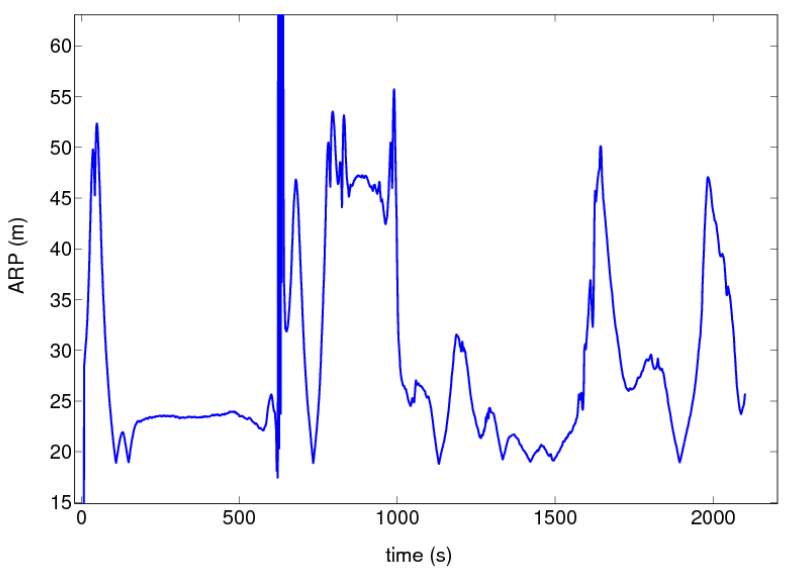

Fig. 3. ARP of the current location on the whole test trajectory

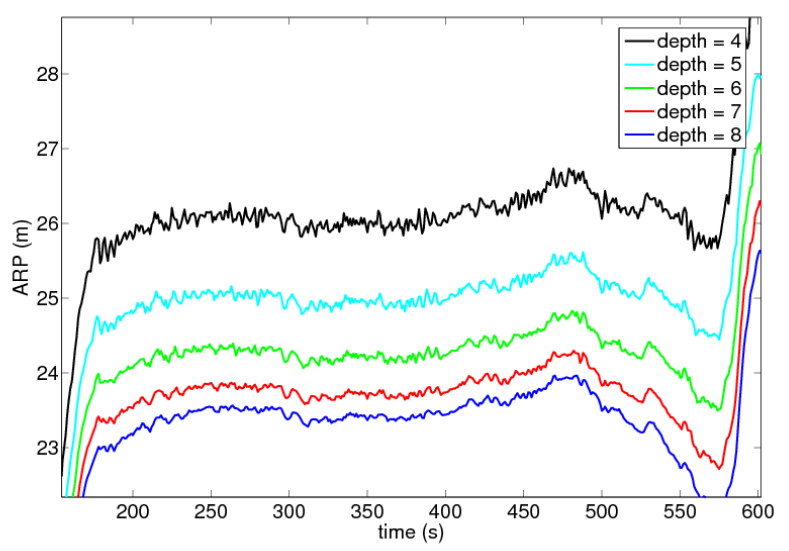

Fig. 4. ARP comparison for different horizon depths

and PL algorithms. It will include use cases with long partial occlusions and simultaneous multi-path.

\section{CONCLUSIONS}

Conventionally, integrity measures of localisation systems have been limited to the aerospace domain and centered on GPS measurements, in this paper through a new formulation that incorporates vehicle ego-state data, it was possible to extend them to mobile ground robotic applications. The proposed approach uses past information and provides an adapted formulation for FDE and PL algorithms. FDE algorithms are therefore applied not only to GPS measurements but include vehicle ego-state measurements and the evolution model. Another advantage is the reduction of the PL with the help of these new data. This formulation increases data redundancy availability and therefore extends the usage of FDE and PL algorithms throughout most of the vehicle trajectory.

The experimental results have shown that it is possible to compute the ARP and to detect faults for outdoors mobile platforms using the proposed formulation. The algorithms perform well when moving at most speeds, except when the vehicles were at very low speeds. This is due to the proximity of the positions considered as part of the data horizon. It leads to singularities affecting the computation when a temporal sampling is used. The problem will be addressed by applying a spatial sampling.

The increase of redundancy could provide opportunities to apply non standard FDE algorithms like RANCO [15]. Adding further constraints like the geometry of the roads available from accurate digital maps is another possibility opened by the formulation, it will be possible to enhance the integrity estimations. Information, which will lead to the use of localisation based solutions for safety-critical applications like guiding intelligent passenger vehicles.

\section{REFERENCES}

[1] S. Feng and W. Ochieng, "Integrity of navigation system for road transport," in Proc. 14th World Congress of Intelligent Transportation Systems, Oct. 2007.

[2] H. Durrant-Whyte, D. Pagac, B. Rogers, M. Stevens, and G. Nelmes, "An autonomous straddle carrier for movement of shipping containers," IEEE J. Robot. Automat., vol. 14, pp. 14-23, Sept. 2007.

[3] J. Ibaï£;ez-Guzmï£;n, O. Le Marchand, and C. Chen, "Metric evaluation of automotive-type GPS receivers," in Proc. FISITA, Sept. 2008

[4] R. Isermann and P. Balle, "Trends in the applicaiton of modelbased fault detection and diagnosis of technical processes," Control Engineering Pratice, vol. 5, no. 5, pp. 709-719, 1997.

[5] R. Young, G. McGraw, and B. Driscoll, "Investigation and comparison of horizontal protection level and horizontal uncertainty level in FDE algorithms," in Proc. of the Institute of Navigation, International Technical Meeting, Sept. 1996, pp. 1607-1614.

[6] R. G. Brown, "A baseline RAIM scheme and a note on the equivalence of three RAIM methods," in Proc. of the 1992 National Technical Meeting of the Institute of Navigation, Jan. 1992, pp. 127-137.

[7] S. Hewitson and J. Wang, "GNSS receiver autonomous integrity monitoring (RAIM) for multiple outliers," European Journal of Navigation, vol. 4, no. 4, pp. 47-57, 2006.

[8] M. A. Sturza and A. K. Brown, "Comparison of fixed and variable threshold RAIM algorithms," in Proc. of the 3rd International Technical Meeting of the Satellite Division of the Institute of Navigation ION GPS 1990, Sept. 1990, pp. 437-443.

[9] B. Belabbas and F. Gass, "RAIM algorithms analysis for a combined GPS/galileo constellation," in Proc. ION GNSS, Sept. 2005.

[10] S. Hewitson and J. Wang, "GNSS receiver autonomous integrity monitoring (RAIM) performances analysis," GPS Solutions, vol. 10, no. 3, pp. 155-170, July 2006.

[11] S. Sukkarieh, E. M. Nebot, and H. Durrant-Whyte, "A high integrity IMU/GPS navigation loop for autonomous land vehicle applications," IEEE Trans. Robot. Automat., vol. 15, no. 3, pp. 572-578, June 1993.

[12] O. Le Marchand, P. Bonnifait, J. Ibaï£ $; e z-G u z m i ̈ £$ n, F. Peyret, and D. Bï¡taille, "Performance evaluation of fault detection algorithms as applied to automotive localization," in Proc. of the European Navigation Conference GNSS, Apr. 2008.

[13] O. Adrot, D. Maquin, and J. Ragot, "Fault detection with model parameter structured uncertainties," in Proc. European Control Conference, (ECC97), Aug./Sept. 1999.

[14] F. Gustafsson, "Stochastic observability and fault diagnosis of additive changes in state space models," in Proc. IEEE International Conference on Acoustics, Speech, and Signal Processing, vol. 5, 2001, pp. 2833-2836.

[15] G. Schroth, A. Ene, J. Blanch, T. Walter, and P. Enge, "Failure detection and exclusion via range consensus," in Proc. of the European Navigation Conference GNSS, Apr. 2008. 